\title{
Do-It-Yourself Database for Core Facility Management
}

\author{
Ru-ching Hsia ${ }^{1}$
}

${ }^{1}$ Electron Microscopy Core Imaging Facility, University of Maryland Baltimore, Baltimore, USA.

Core facility directors and staff who are often highly trained scientists face the unique challenge of managing the day-to-day operation of the facility [1]. A recent survey reported that core facility managers spend an average of 55 hours per month on administrative tasks [2]. Although several core facility management software packages are available commercially, these programs are often costly and may require the purchase of an annual maintenance contract at a substantial price. Nearly 50\% of the core facilities that responded to the survey did not use an electronic management system specifically designed for core facility management. Here, I present a do-it-yourself database management system that can be custom-designed and implemented by core facility personnel without prior software programming training.

The Electron Microscopy Core Imaging Facility (EMCIF) of the University of Maryland Baltimore is a mid size core facility that serves an active user base of 100, performs 150 to 200 research projects annually and generates 60-100 GB of image data. The EMCIF management database uses Microsoft Office Access, a program included in the Microsoft Office Professional suite. The EMCIF day-to-day records are divided in several main tables such as client information, project information, project charges, project experiments, etc. Records in the tables are safely stored in a web-based server networked to all computers in the facility. Each facility client and project is given a unique identifier which is cross-referenced throughout the database via queries and defined relationships. Many command buttons for performing simple tasks such as opening and printing forms are already included in the Access program. Using these built-in command buttons, invoices, quotes and project summaries can be printed as reports, exported to other programs, or e-mailed to clients with a single click of the mouse.

Figure 1 shows the switchboard that the EMCIF staff use on a daily basis to perform all tasks including conducting service projects, facility management, user communication, billing and purchasing (Figure 1A, B C and D). The EMCIF database was initially constructed in 2009 with five tables to manage service records. It has been expanded and updated regularly based on staff feedback and growth of the facility. The database now holds records for approximately 1000 projects and more than 300 clients; each record can be retrieved using custom-built search engines within seconds (Figure 1D). This database allows the EMCIF director and staff members to track down information concerning a given EMCIF project workflow, spending or income in real time. Data entry is simplified by specific entry forms designed for a given task with built-in drop-down menus to minimize typing (Figure 2). A significant advantage of this 'DIY' database is its ease to adapt or expand in order to fit EMCIF and clients' changing needs, an option often not available in commercial software. Web-based surveys or registration forms can be seamlessly linked to the record table stored on the server, thus easing the task of collecting client information. Finally, it is also worth noting that many resources such as Access Database Template, tutorials and user forums are available to assist in the construction of a core facility management database that is tailored to the facility's needs.

\section{References:}


[1] H Wallrabe, A Periasamy and M Elangovan, Microscopy Today 22 (2014), p.36. [2] iLabSolutions, The 2014 Core Facility Benchmarking Study.
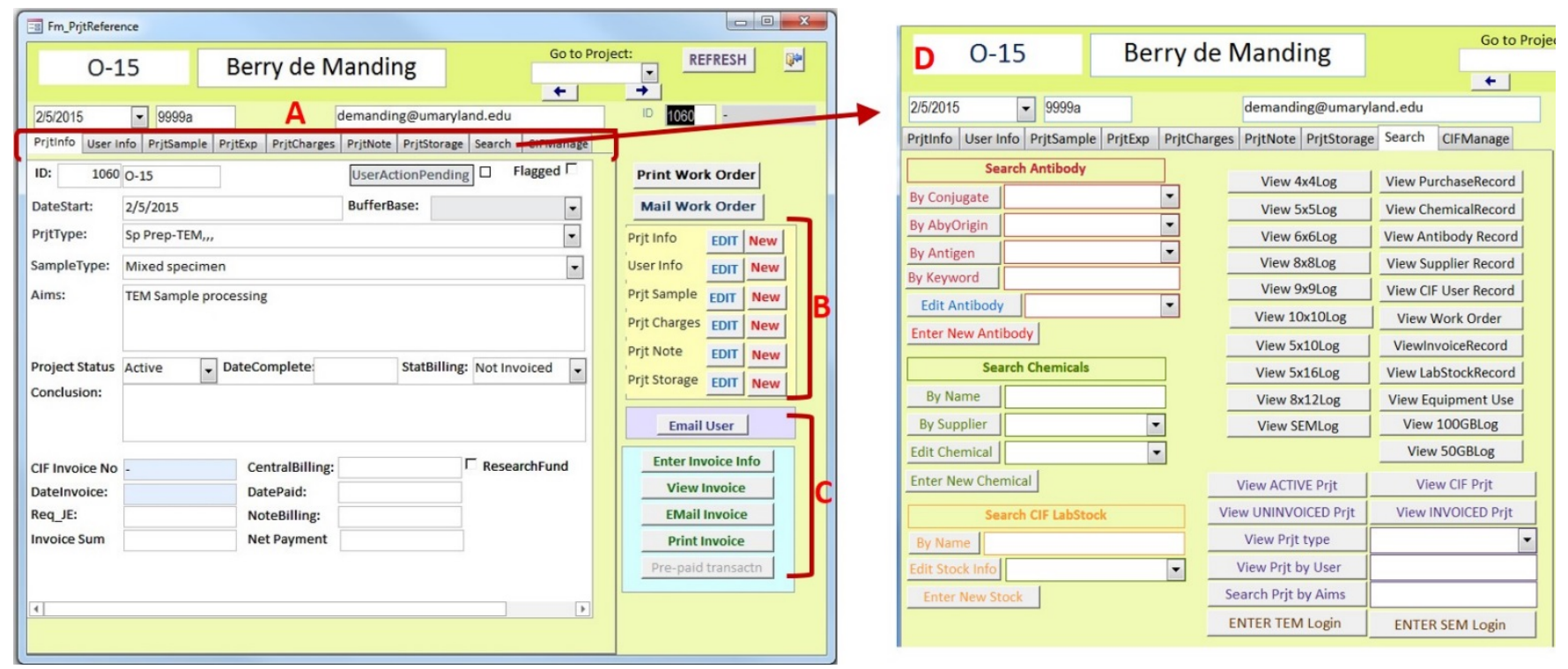

Figure 1. The switchboard of EMCIF database allows staff to keep track of all day-to-day tasks. The switchboard is divided into submenus (A), command buttons for data entry (B) and command buttons to generate reports and invoices (C). Search page with built-in search engine to retrieve or view data by keywords or specific criteria (D) are also accessible from the switchboard.
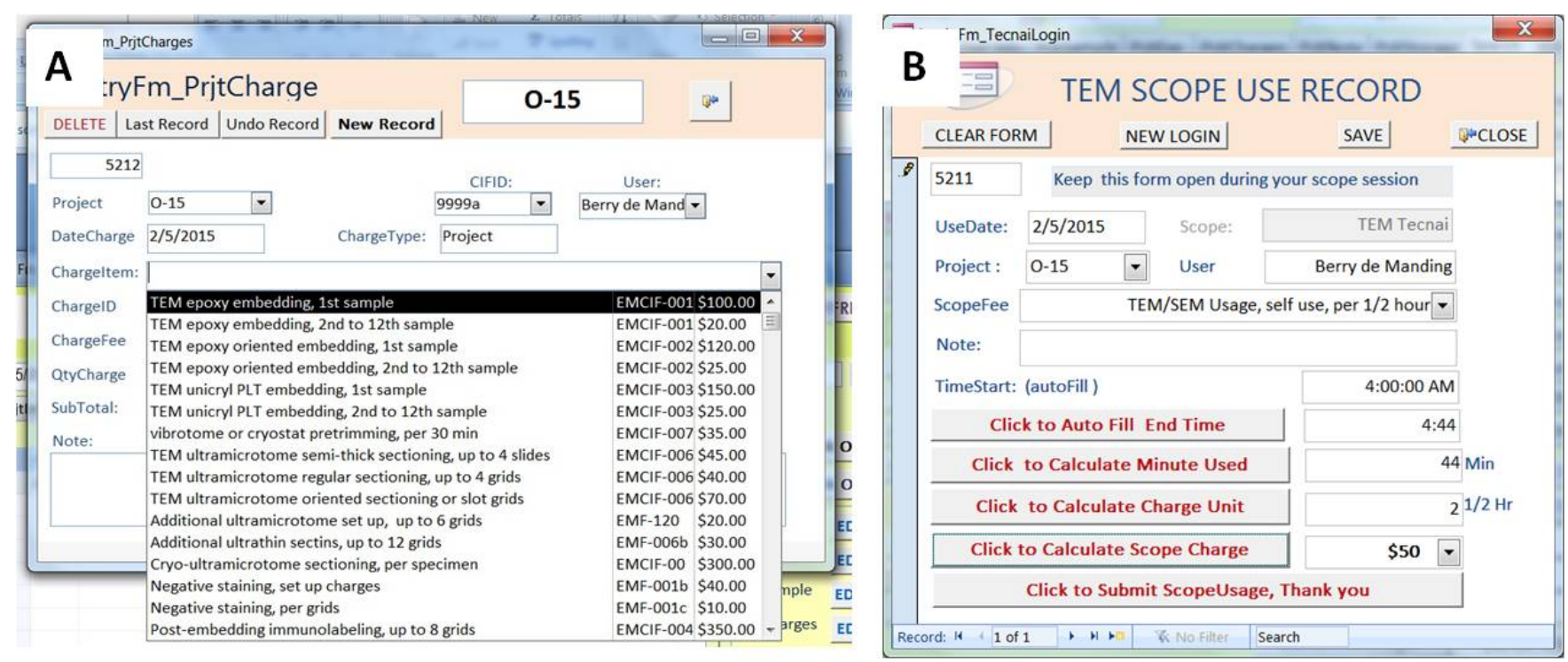

Figure 2. Different entry forms are used for data entry of different tasks. Data for the same project can be entered simultaneously from different location and by different personnel. For instance, service charges for project O-15 are entered by EMCIF staff in project charge entry form (A). Scope time used by the client, Berry de Manding for project O-15 is entered directly from the scope PC to the database by using scope use entry form (B). 